AIP Conference Proceedings Volume 2000, 7 August 2018, Article number 0200193, 7th Meeting of Departments of Fluid Mechanics and Thermodynamics; Hotel Thermal Sturovo; Slovakia; 27 June 2018 through 30 June 2018; Code 138542

\title{
On Reynolds Number Physical Interpretation
}

\author{
Václav Uruba ${ }^{1,2}$ a) \\ ${ }^{1}$ University of West Bohemia, Univerzitni 22, Pilzen, CR \\ ${ }^{2}$ Institute of Thermomechanics CAS, v.v.i., Dolejškova 5, Praha 8, CR \\ a)uruba@kke.zcu.cz
}

\begin{abstract}
The physical interpretation of Reynolds number in laminar and turbulent flows is studied in details. Understanding to its real physical meaning is the necessary condition for proper and meaningful explanation of the processes behind. The Reynolds number is interpreted in the paper as ratio of momentum convection and momentum diffusion effects in a laminar-like flow.
\end{abstract}

\section{INTRODUCTION}

The concept of Reynolds number (hereinafter Re) was introduced by Sir George Stokes in 1851, but this dimensionless parameter was given its name by Arnold Sommerfeld in 1908 after Osborne Reynolds (1842-1912), who popularized its use in 1883 in his famous experimental study on a pipe flow transition to turbulence [2].

Over last more than hundred years it was proved by both theory and practice, that the Reynolds number plays fundamental role in fluid dynamics, taking into account viscose and inertial effects. Reynolds number is the parameter determining the flow quality. However the physical interpretation of the Reynolds number is not straightforward and could be misleading.

Reynolds number is interpreted as ratio of inertial and viscous forces as presented in many textbooks (see e.g. [3]). This traditional interpretation suffers from logical justification, as the forces should be in equilibrium any time. Better, the Reynolds number could be considered as ratio of inertial and viscose effects. However this interpretation is meaningful only in laminar flow.

Anyway, the Reynolds number definition makes sense only within shear layers, where both viscose and inertial effects are present. Outside the shear flow regions the viscous forces vanish and the Reynolds number is thus undefined. The same statement holds for inviscid fluid flow anywhere.

In turbulence the interpretation is very different. Turbulent flow occur at high Re, it originates as an instability of a laminar flow if the Re becomes too large. The instabilities are related to the interaction of viscous terms and nonlinear inertia terms in the equations of motion. This is a very complex process involving randomness, theory of partial differential equations is not developed in this area. The turbulent Re is defined using velocity standard deviation (i.e. square root of turbulent kinetic energy) and size of the vortices as a rule. Then, the Reynolds number represents ratio of turbulent and molecular viscosity. There are a few very different, however physical interpretations of the Reynolds number in turbulence.

For example Lighthill [1] interprets Reynolds number in turbulence by analysis of the turbulent kinetic energy equation as the ratio of turbulent energy production per unit mass $U^{3} / L$ to the corresponding rate of viscous dissipation $v U^{2} / L^{2}$. The scales are related to the typical eddies, here. Lumley in his classic textbook on turbulence [4] defines physical meaning of the Re as ratio of length-scales or time-scales in the turbulent flow. Reynolds number of a turbulent flow may be interpreted as a ratio of a turbulence time-scale $L / U$ to a molecular time-scale $L^{2} / v$ that would prevail in the absence of turbulence in a problem with the same length-scale. Lumley states that this point of view is more reliable than thinking of $\mathrm{Re}$ as a ratio of inertia to viscous effects in the governing equations. That could 
be misleading as especially at high Re viscous and other diffusion effect tend to operate on smaller length scales than inertia effects.

The presented paper is devoted to definition of the Reynolds number and explanation of its meaning in laminarlike flow in shear layers.

\section{REYNOLDS NUMBER DEFINITION}

The definition of the Reynolds number is given by the following well-known formula:

$$
\operatorname{Re}_{L}=\frac{U L}{v}
$$

$U$ holds for a typical velocity, $L$ is a typical length and $v$ is kinematic viscosity.

Please note that the numerator consists of quantities defining the flow and the denominator is represented by the fluid property only.

Usually, the typical velocity and fluid viscosity are defined unambiguously. Kinematic viscosity is the fluid property given by the fluid nature and its state (static pressure and temperature). The typical velocity is usually chosen as velocity range within the shear region very often. For example, in the case of a boundary layer the velocity ranges from 0 on the wall to velocity of outer stream, so the typical velocity is the outer stream one.

However, there is a problem in definition of the typical length. Sometimes it is represented by a scale in the streamwise direction (e.g. airfoil chord) in other cases the spanwise length parameter is used (e.g. boundary layer thickness or pipe diameter), sometimes a characteristic dimension of the rigid boundary is considered with no simple link to the flow-field (e.g. cylinder diameter in cross-flow). Obviously, the choice of the length parameter used in the Reynolds number definition is determining its physical meaning.

Its general form could be derived with help of dimensional analysis of the flow dynamics. For the case of viscose incompressible fluid-flow without presence of other volume forces the Reynolds number is the only relevant parameter defining quality of the flow under given boundary conditions (see e.g. [5]). It determines presence of coherent structures in the flow and their behavior. And of course also the type of the flow: laminar or turbulent.

\section{REYNOLDS NUMBER IN LAMINAR BOUNDARY LAYER}

The laminar boundary layer flow is considered. Prandtl's theory of boundary layer could be adopted to simplify the Navier-Stokes (NS) equations, which govern the case. The details could be find in any good fluid mechanics textbook, e.g. [3]. The schematic view of the boundary layer is shown in fig. 1.

The final equations of motion for steady two-dimensional incompressible flow in a laminar boundary layer after omitting the small terms of higher order are as follows:

$$
\begin{aligned}
\frac{\partial u}{\partial x}+\frac{\partial v}{\partial y} & =0 \\
u \frac{\partial u}{\partial x}+v \frac{\partial u}{\partial y} & =-\frac{1}{\rho} \frac{\partial p}{\partial x}+v \frac{\partial^{2} u}{\partial y^{2}} \\
0 & \approx-\frac{1}{\rho} \frac{\partial p}{\partial y}
\end{aligned}
$$

The first equation is the continuity, the other 2 equations are the momentum (NS) in $x$ (streamwise) and $y$ (spanwise, perpendicular to the wall) directions respectively. Boundary conditions include vanishing both velocity components $u$ and $v$ on the rigid wall and velocity $U$ of the outer stream. 


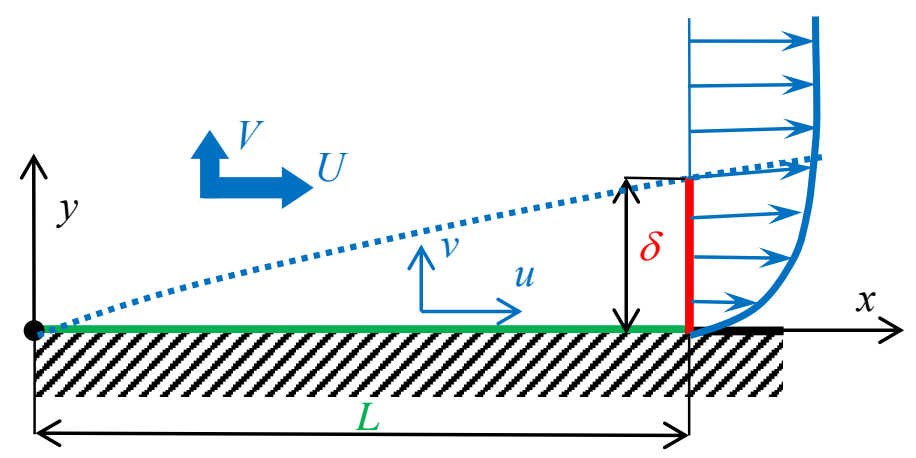

Figure 1. Scheme of laminar boundary layer

Now, let us consider the flow-field not too close to the surface. In such situation the flow contains the lengthscale $L$ determined by the geometry of the body and convection process, e.g. for an airfoil the chord length would be characteristic. The second length-scale is the boundary layer thickness $\delta$, it is characterizing the momentum diffusion process due to molecular mechanism.

From the continuity equation we could estimate $\partial u / \partial x \approx U / L$, while $\partial v / \partial y \approx V / \delta$ and we end-up with estimate of the velocity component perpendicular to the wall on the boundary layer border $V \approx \delta U / L$.

The $y$-momentum equation indicates constant pressure across the boundary layer. If zero pressure gradient along the boundary layer is present, then the pressure is constant in the whole domain. Now, both nonlinear inertial terms are of the order $u \partial u / \partial x \approx v \partial u / \partial y \approx U^{2} / L$, while the viscose term is of order $v U / \delta^{2}$. Within the inertial terms the streamwise length-scale $L$ is present, while in the viscose term the typical length-scale is the boundary layer thickness $\delta$. The $x$-momentum equation now yields:

$$
\frac{U^{2}}{L} \approx \frac{v U}{\delta^{2}}
$$

Please note, that the $x$-momentum equation states clearly in the constant pressure case that the viscous force is equal to the inertial force for any velocity value, so the ratio of inertial and viscose forces is always equal to 1 for the laminar boundary layer without pressure gradient!

From the formula (3) we could derive Reynolds number, defined with help of $L$ or $\delta$ :

$$
\operatorname{Re}_{L}=\frac{U L}{v} \sim\left(\frac{L}{\delta}\right)^{2}, \quad \operatorname{Re}_{\delta}=\frac{U \delta}{v} \sim \frac{L}{\delta}
$$

So, the Reynolds number $\operatorname{Re}_{\delta}$ could be interpreted as ratio of convective and diffusive length-scales of the flow within the shear layer, $L$ and $\delta$.

On the other hand, the Reynolds number $\operatorname{Re}_{L}$ is proportional to the square of the same ratio.

Obviously, the $\operatorname{Re}_{L}$ is proportional to square of the $\mathrm{Re}_{\delta}$. So, the two Reynolds numbers represent themselves two completely different parameters with different physical meaning.

From the Blasius solution of a laminar boundary layer, the thickness of the boundary layer $\delta$ could be evaluated precisely, then the solution gives:

$$
\frac{\delta}{L}=\frac{C}{\sqrt{\operatorname{Re}_{L}}}=\frac{C^{2}}{\operatorname{Re}_{\delta}},
$$


where $C$ is constant, its value depends on the boundary layer thickness definition: $C=4.91$ for conventional $99 \%$ BL thickness, $C=1.72$ for displacement BL thickness and $C=0.664$ for impulse (momentum) BL thickness respectively (see e.g. [3]).

\section{CONCLUSION}

The Reynolds number is the ratio of scales in flow, the scales could be defined as time-scales or length-scales. Then, the Reynolds number physical meaning is the ratio of advection (due to convection) scale to dissipation (molecular viscosity related) scale. The flow region over which the Reynolds number is defined is considered as laminar flow - the whole region of the laminar flow-field, or laminar-like eddy within the turbulent flow.

More generally, the Reynolds number could be understood as the ratio of inertial and viscous effects, or, more precisely, the ratio of momentum convection and momentum diffusion length-scales.

\section{ACKNOWLEDGMENTS}

This work was supported by the Technology Agency of the Czech Republic, EPSILON programme, project TH02020057.

\section{REFERENCES}

1. M. J. Lighthill, "An informal introduction to theoretical fluid mechanics," Oxford: Clarendon Press, 1986.

2. O. Reynolds, "An Experimental Investigation of the Circumstances Which Determine Whether the Motion of Water Shall be Direct or Sinuous, and the Law of Resistance in Parallel Channels," (Philosophical Transactions of the Royal Society of London 174, 1883), pp. 935-982.

3. B. R. Munson, A. P. Rothmayer, T. H. Okiishi, "Fundamentals of Fluid Mechanics," 7th Edition, Wiley, 2012.

4. H. Tennekes, J. L. Lumley, "A first course in turbulence," The MIT Press, 1972.

5. V. Uruba, "Turbulence," textbook CTU Praha, (in Czech and English) 2009, 2014. 